\title{
An Ensemble Downscaling Prediction Experiment for Medium-Range Forecast of the Daily Mean Surface Temperature Distribution over Northeastern Japan during Summer
}

\author{
Shin FUKUI, Toshiki IWASAKI, and Weiming SHA \\ Graduate School of Science, Tohoku University, Sendai, Japan
}

(Manuscript received 14 November 2013, in final form 29 August 2014)

\begin{abstract}
To assess medium-range forecasts of detailed spatial distributions of the daily mean temperature, an ensemble downscaling forecast experiment was conducted using the Japan Meteorological Agency (JMA) nonhydrostatic model (NHM) with horizontal resolutions of $25 \mathrm{~km}$ and $5 \mathrm{~km}$. Special attention was paid to the anomalously cool summers over northeastern Japan caused by northeasterly winds called Yamase. The results are validated against the daily mean surface temperatures observed by the Automated Meteorological Data Acquisition System (AMeDAS) in the study area.

Ensemble mean downscaling forecasts can successfully extract reliable signals with information about local circulations. The ensemble mean forecasts reduce the root mean square errors of the daily mean surface temperature by $15 \%$ compared to single downscaling forecasts. The ensemble spreads also indicate the possibility of making probabilistic predictions that consider the effects of local circulations in addition to large-scale motions. The ensemble downscaling forecasts have $80 \%$ larger spreads than the global forecasts with the JMA global spectral model at a resolution TL159L60 and approach the theoretical value.

An empirical orthogonal function (EOF) analysis indicates that the predictability depends on the EOF modes. The predictable periods are 8 days for the homogeneous mode over northeastern Japan, 5 days for the Yamase mode (east-west mode), and 2 days for the north-south mode. The dynamical downscaling can properly predict the amplitudes of the EOF modes. In particular, the dynamical downscaling can predict $90 \%$ of the Yamase mode, as compared to $20 \%$ prediction of the global model for the same mode.
\end{abstract}

Keywords dynamical downscalong; ensemble forecast; local temperature distribution; Yamase wind

\section{Introduction}

Northeasterly winds called Yamase brings anomalously cool and cloudy weather over northeastern Japan during the summer and cause serious damage to crops. Yamase itself is considered as a largescale flow from a cool polar maritime air mass that

Corresponding author: Shin Fukui, Graduate School of Science, Tohoku University, 6-3 Aramaki, Aza-Aoba, Aoba-ku, Sendai, Japan

E-mail: fukui@wind.gp.tohoku.ac.jp

(C)2014, Meteorological Society of Japan develops over the Okhotsk Sea, the Bering Sea and the North Pacific (Ninomiya and Mizuno 1985a). Thus, it has been an important feature of medium-range forecasts (3-10 days) in Japan. The vertical scale of Yamase is less than $1-2 \mathrm{~km}$; thus, it is greatly influenced by the mountains that run from north to south over northeastern Japan, and it causes a characteristic temperature distribution that includes a large temperature contrast between the eastern and western sides of the region (Ninomiya and Mizuno 1985b) as shown in Fig. 1. The prediction of these spatially-detailed temperature fields is helpful for reducing the 


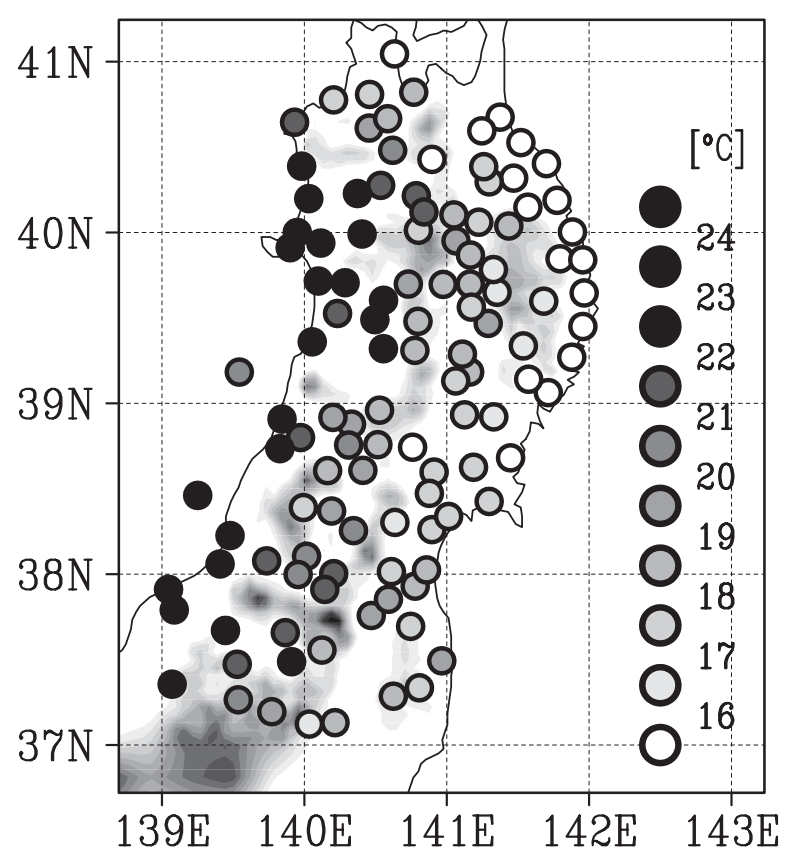

Fig. 1. The daily mean surface temperature distribution observed at AMeDAS sites on a typical Yamase day (24 June 2003) over northeastern Japan

damage caused by Yamase. These features must be simulated with high resolution models.

Dynamical downscaling is a technique that is used to derive information about small-scale phenomena from large-scale conditions using high-resolution regional models. The method can explicitly take into account small-scale phenomena such as complex terrain and local circulation in its area of interest. Ishizaki et al. (2012) downscaled the Japanese 25year reanalysis (JRA-25; Onogi et al. 2007) with five different models with horizontal resolutions of 20 $\mathrm{km}$. They showed that dynamical downscaling can improve the reproducibility of the temperature distributions that are influenced by Yamase over northeastern Japan using the monthly averaged temperature anomaly fields in July 1993.

Dynamical downscaling data include errors that arise from uncertainties in the initial and lateral boundary conditions and deficiencies of the downscaling model. Dynamical downscaling forecasts primarily depend on the lateral boundary conditions especially in small domains (Vukicevic and Errico 1990). The lateral boundary conditions are generally provided by global forecasts or by data derived from them in order to predict the future state of the atmo- sphere with dynamical downscaling. The performance of global forecasts is mainly affected by uncertainties of the initial conditions. Several ensemble forecast systems have been developed to consider the uncertainties of the initial conditions, and the implementation of these ensemble approaches has improved forecasts and enabled probabilistic predictions (e.g., Toth and Kalnay 1993; Buizza and Palmer 1995; Wei et al. 2008). Ensemble downscaling forecasts are expected to enable us to consider the influences of these uncertainties and extract more reliable information about local circulations in addition to largescale motions. Several studies have investigated the benefits of ensemble downscaling in medium-range rainfall forecasts. Marsigli et al. (2001) showed that ensemble downscaling forecasts efficiently captured four heavy rainfall events over the Alpine than global ensemble forecasts or deterministic limited-area forecasts. Marsigli et al. (2008) indicated that ensemble downscaling forecasts are more useful than global ensemble forecasts in predicting the occurrence of precipitation peaks and distinguishing between events and non-events, particularly for moderate and high precipitation.

Takai et al. (2006) extracted the eigenmodes that represent Yamase from an empirical orthogonal function (EOF) analysis of observed surface temperature fields over northeastern Japan. It is expected that the modes obtained from EOF analysis will be useful in evaluating predictability of the fields.

The purpose of this study is to assess the ensemble downscaling system by focusing on medium-range forecasts of daily mean surface temperature distributions over northeastern Japan and to investigate the predictability of the modes obtained by the EOF analysis of observed daily mean surface temperature fields.

The outline of the paper is as follows. In Section 2 , we describe the design of the experiment and the validation method. We show the effectiveness of ensemble downscaling in forecasting surface temperature in Section 3. The predictability is investigated in terms of the EOF modes from the surface temperature fields in Section 4. We discuss the difference between the results obtained from the global and downscaling forecasts in Section 5. The conclusions are summarized in Section 6.

\section{Experimental setup}

\subsection{Ensemble downscaling forecast}

In this study, the Japan Meteorological Agency (JMA) nonhydrostatic model (NHM; Saito et al. 2007) is used as the dynamical downscaling model. 
(a) NHM-25km

(b) NHM-5km

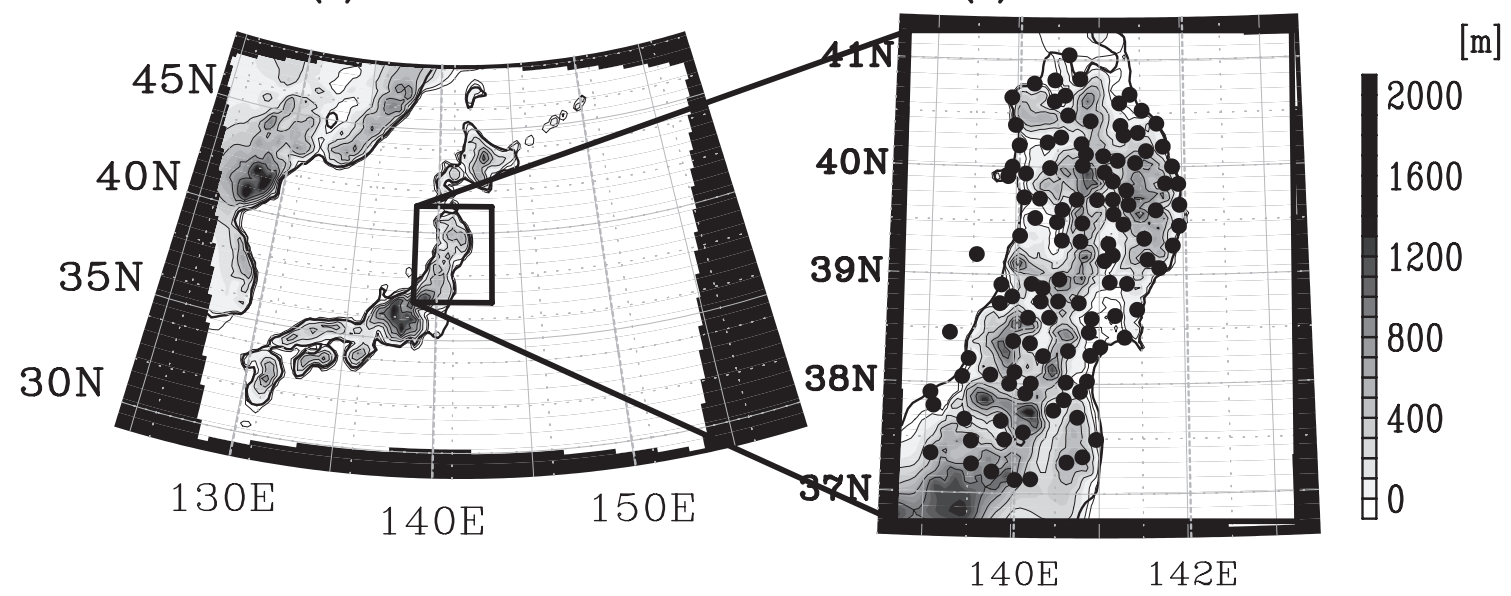

Fig. 2. The domains for downscaling at (a) $25 \mathrm{~km}$ horizontal resolution and (b) $5 \mathrm{~km}$ horizontal resolution. The black dots represent the AMeDAS sites.

The ensembles consist of nine members. The initial and lateral boundary conditions for each member are taken from one-month global ensemble hindcasts produced by JMA. The one-month global ensemble hindcasts are integrated from 9 different initial conditions at each initial time with the JMA global spectral model (GSM; JMA 2013) at a resolution TL159L60 and interpolated to $1.25^{\circ}$ latitude-longitude grids. The initial conditions for one of the nine runs are the JRA-25 or JMA Climate Data Assimilation System (JCDAS) at the initial time, and the initial conditions for the other eight runs are created by adding perturbations created using breeding method (Toth and Kalnay 1997) to JRA-25/JCDAS at the initial time. The sea surface temperature (SST) at the initial time is taken from the centennial in situ observation based estimates of variability of SST and marine meteorological variables (COBE-SST; Ishii et al. 2005) on that day. The anomalies from the COBE-SST climatology (19792004) on the initial day are fixed during the forecast time. The set of parameterizations for the physical process in GSM is as follows (JMA 2013). Prognostic Arakawa and Schubert scheme (Arakawa and Schubert 1974) is used for convective parameterization. The cloud amounts and cloud water contents are obtained from a simple statistical approach proposed by Sommeria and Deardorff (1977) but by using a uniform probability function. The cloud amount of marine stratocumulus is diagnosed using the scheme of Kawai and Inoue (2006). The Mellor-Yamada level 2 scheme (Mellor and Yamada 1974) is used as the turbulent closure model. The simple biosphere scheme (Hirai et al. 2007) is adopted as the land surface model. The radiative transfer equation is computed every three hours for longwave radiation and every hour for shortwave radiation. The parameterization for gravity-wave drag (Iwasaki et al. 1989) is adopted.

A double-nesting approach is used in this downscaling experiment. The horizontal resolutions are 25 $\mathrm{km}$ with $100 \times 80$ grid points in the first stage, and $5 \mathrm{~km}$ with $80 \times 100$ grid points in the second stage. Hereafter, the downscalings with $25 \mathrm{~km}$ and $5 \mathrm{~km}$ horizontal resolution NHMs are denoted as NHM-25km and NHM-5km, respectively. The domains for this study are shown in Fig. 2. The model topography is created from GTOPO30 data. The time intervals are 40 seconds in NHM-25km and 20 seconds in NHM- $5 \mathrm{~km}$. To suppress computational noise, the larger damping parameters are used in NHM- $5 \mathrm{~km}$. To conduct a long-term integration that is consistent with the large-scale fields provided by the global model, the spectral boundary coupling method (Kida et al. 1991; Yasunaga et al. 2005) is applied above 7 $\mathrm{km}$. The vertical layer is composed of 40 levels from $20 \mathrm{~m}$ to $23,205 \mathrm{~m}$ in the generalized hybrid vertical coordinates. The Kain-Fritsch scheme (Kain and Fritsch 1990) is used for the convective parameterization, and the cloud microphysics scheme is a six-class bulk microphysics scheme (Ikawa and Saito 1991). The Mellor-Yamada level 3 improved by Nakanishi and Niino $(2004,2006)(\mathrm{MYNN})$ is adopted as the turbulent closure model. Nagasawa et al. (2006) 
described that a partial condensation scheme is necessary to accurately deal with the effects of the low-level clouds that are accompanied by Yamase in models coarser than $100 \mathrm{~m}$, which can not explicitly resolve shallow convection. The partial condensation scheme (Sommeria and Deardorff 1977) is employed to estimate subgrid-scale condensation based on subgrid-scale inhomogeneities derived from MYNN and diagnosed cloud amounts for radiation estimation. $\mathrm{SiB}$ (Hirai et al. 2007) is adopted as the land surface model. The radiative transfer equation is computed every 10 minutes for both longwave and shortwave radiation. The SST at the initial time is taken from the National Oceanic and Atmospheric Administration's daily optimum interpolation SST analysis data (OISST: Reynolds et al. 2007) on that day. The anomalies from the OISST climatology (2000-2009) data on the initial day are fixed during the forecast time.

Considering that Yamase occurs most frequently between late June and mid-July (Kanno 1993), the initial times for NHM-25km are set to 12 UTC on 20 June, 30 June, 10 July, and 20 July from 2000 to 2009. The initial times for NHM-5km are set to 15 UTC. The forecast period is 14 days.

\subsection{Validation}

To assess the performance of the ensemble downscaling forecast system, the predicted daily mean surface temperature fields are verified in terms of the root mean square errors (RMSEs) and ensemble spreads. The daily mean value is defined as the average of the values at $03,09,15$, and $21 \mathrm{LT}$ in Japan, which is 9 hours ahead of UTC, based on the availability of the hindcast data set. The RMSEs are evaluated against the daily mean temperature data observed at 117 surface sites of the Automated Meteorological Data Acquisition System (AMeDAS) operated by JMA. The AMeDAS sites are located in the domain of NHM- $5 \mathrm{~km}$ and contain no missing values during the experimental periods (Fig. 2b). Note that the AMeDAS data are assumed to have no errors in this study. The global forecasts and the downscaling forecasts are linearly interpolated horizontally to each AMeDAS site. There is a mismatch between the interpolated points and the corresponding AMeDAS sites in the vertical direction. In addtion, the forecasts contain biases derived from model deficiencies. Considering these factors, the biases are defined as

$$
b_{i, t}=\frac{1}{N} \sum_{k=1}^{N}\left(x_{i, t, k}-y_{i, t, k}\right) \text {, }
$$

where $x_{i, t, k}$ indicates the forecasts that are horizontally interpolated to site $i$ on a forecast lead time $t$ days from the initial date $k, y_{i, t, k}$ indicates the corresponding AMeDAS observation, and $N$ denotes the total number of intial dates. Because we focus on the anomaly components from climatology, the biases are corrected at each AMeDAS site at each forecast lead time. To assess predictions, it might be better to estimate the biases during different training periods from the experimental periods. However, we did not do this because the computational resources are limited and the number of cases is considered to be large enough to ignore the influence of one specific case. The bias correction can reduce the RMSEs of NHM-25km and NHM- $5 \mathrm{~km}$ by an average of $30 \%$.

\subsection{EOF mode decomposition}

To investigate the predictable signals for medium-range forecasts, we decomposed the ensemble mean forecasts and the AMeDAS observations of the daily mean surface temperature fields into the eigenmodes from the EOF analysis of the AMeDAS observations of the temperature fields. The procedure is as follows. The component of the climatological seasonal march at site $i$ on date $d$ corresponding to the forecast lead time $t$ for the initial date $k$ is assumed as

$$
s_{i, d}=y_{i, d}^{\mathrm{clm}}-\bar{y}_{i}^{\mathrm{clm}},
$$

where $y_{i, d}^{\text {clm }}$ is the daily climatology evaluated at site $i$ on date $d$ from the AMeDAS observations between 1980 and 2009, which is obtained by passing them through a Lanczos filter (Duchon 1979). $\overline{y^{\mathrm{clm}}}{ }_{i}$ represents the average at site $i$ over the experimental period (21 June-4 August, 2000-2009). The forecast anomaly of the daily mean surface temperature and the corresponding AMeDAS observation anomaly are obtained as

$$
\begin{aligned}
& x_{i, t, k}^{\text {anom }}=x_{i, t, k}-b_{i, t}-\bar{y}_{i}-s_{i, d}, \\
& y_{i, t, k}^{\text {anom }}=y_{i, t, k}-\bar{y}_{i}-s_{i, d},
\end{aligned}
$$

where $x_{i, t, k}$ and $y_{i, t, k}$ are the forecast and AMeDAS observation, respectively, at site $i$ on a forecast lead time of $t$ days for initial date $k$, and $y_{i}$ is the average of the AMeDAS observations at site $i$ over the experimental period. The EOF analysis is applied to the AMeDAS anomaly $y_{i, t, k}^{\text {anom }}$ to extract the EOF modes. Then, $x_{i, t, k}^{\text {anom }}$ and $y_{i, t, k}^{\text {anom }}$ are projected onto the $j$-th EOF mode $\boldsymbol{m}^{j}$. The obtained EOF mode components of the forecasts $C_{x}^{j}$ and that of the AMeDAS observa- 
Natural variability of $\mathrm{T}$ (Single run)

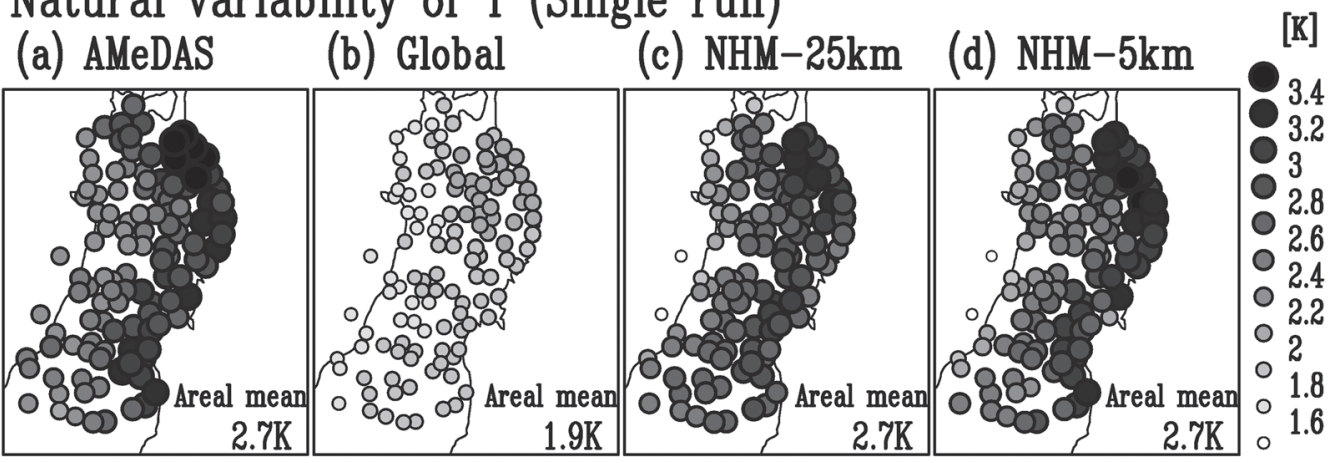

Fig. 3. The natural variability of the daily mean surface temperature averaged over the experimental periods from (a) the AMeDAS observations, (b) the single global single forecasts, (c) the single NHM-25km forecasts, and (d) the single NHM-5km forecasts.

tions $C_{y}^{j}$ are

$$
\begin{aligned}
& C_{x}^{j}=\sum_{i=1}^{L} m_{i}^{j} x_{i, t, k}^{\mathrm{anom}}, \\
& C_{y}^{j}=\sum_{i=1}^{L} m_{i}^{j} y_{i, t, k}^{\mathrm{anom}},
\end{aligned}
$$

where $L$ denotes the total number of the AMeDAS sites. The EOF mode components of the forecasts are compared to those of the AMeDAS observations.

\section{Dynamical downscaling forecast}

\subsection{Natural variability}

We investigate how realistically the natural variability can be reproduced by the models. The natural variability $V_{i, t}$ is defined as

$$
V_{i, t} \equiv \sqrt{\frac{1}{N} \sum_{i, k}^{N}\left(x_{i, t, k}-\frac{1}{N} \sum_{k}^{N} x_{i, t, k}\right)^{2}}
$$

where $x_{i, t, k}$ is the forecast or AMeDAS observation at site $i$ at lead time $t$ days for the forecast running from the initial date $k$, and $N$ denotes the total number of initial dates. Figure 3 shows the natural variability distributions of the AMeDAS observations and the single forecasts. The natural variabilities of the AMeDAS observations are more than $3 \mathrm{~K}$ on the Pacific coast and 2-2.5 K on the Sea of Japan coast. For the global forecasts the natural variabilities are less than $2 \mathrm{~K}$ over the domain. The global forecasts cannot suffciently capture the magnitudes at each station or the contrast between the Pacific coast and the coast of the Sea of Japan. However, for the NHM-25km and NHM-5km forecasts, the natural variabilities are more than $3 \mathrm{~K}$ on the Pacific coast and 2-2.5 K on the coast of the Sea of Japan. The natural variabilities of the NHM- $25 \mathrm{~km}$ and NHM- $5 \mathrm{~km}$ forecasts are comparable to those of the AMeDAS observations at all sites in the domain. Figure $4 \mathrm{a}$ shows the ratio of the natural variability averaged over the sites of the single forecasts to that of the AMeDAS observations. Note that the single forecasts indicate the global control forecasts without the initial perturbations and their downscaling results. The natural variability of the global forecasts represents only $60 \%$ of that of the AMeDAS observations. The underestimation occurs because the global model is too coarse to explicitly simulate smallscale effects, such as local circulations. However, NHM- $25 \mathrm{~km}$ and NHM-5km forecasts have similar variabilities as the observed natural variability. These results indicate that dynamical downscaling is capable of realistically reproducing the natural variability in terms of both magnitude and distribution.

\subsection{Ensemble mean forecasts}

In this subsection, we demonstrate the effectiveness of the ensemble mean in downscaling forecasts. Figure 5a shows the RMSEs of the single forecasts of daily mean surface temperature averaged over the AMeDAS sites and forecast runs. The RMSEs of the NHM-25km and NHM-5km forecasts are $20 \%$ higher than those of the global forecasts. Because the downscaling model can realistically estimate the natural variability, as was described in the previous subsection, single dynamical downscaling forecasts include both smaller-scale and large-scale 
(a) Single run $[K]$

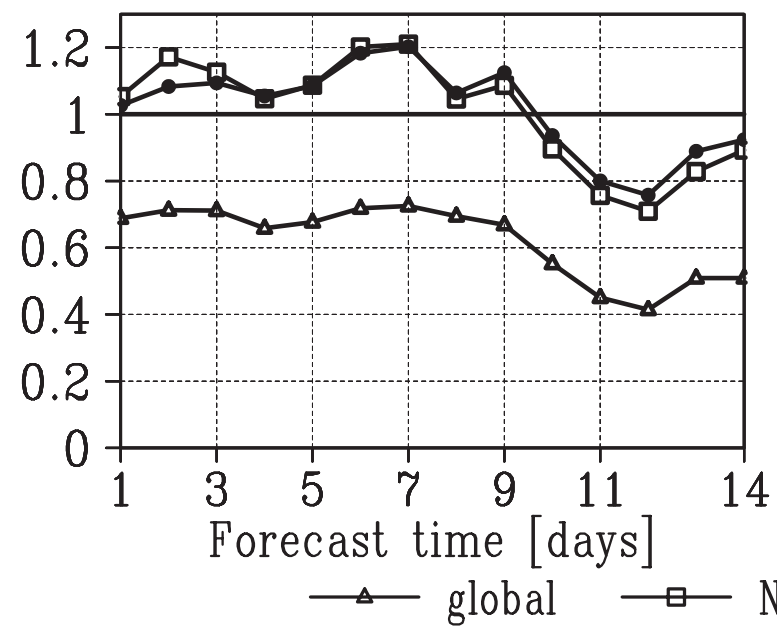

(b) Ensemble mean $[\mathrm{K}]$

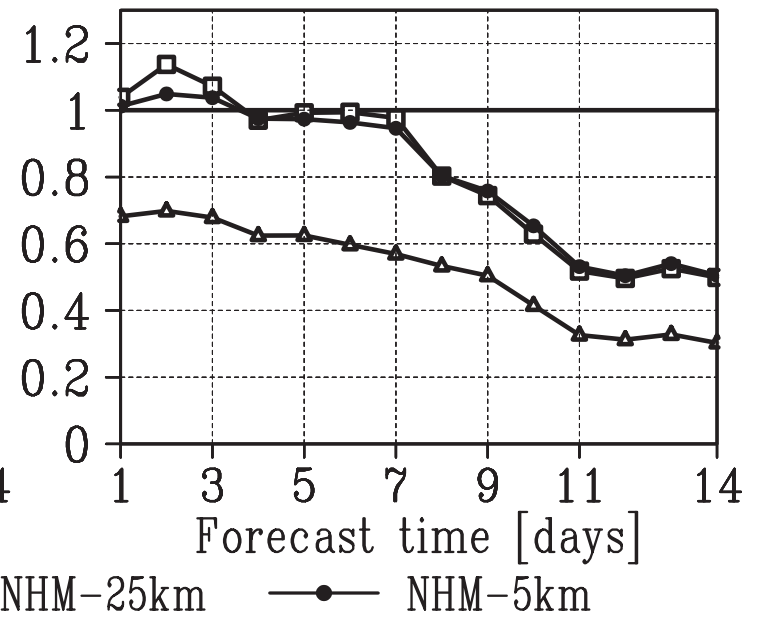

Fig. 4. The ratios of the natural variability of the daily mean surface temperature from (a) single forecasts and (b) ensemble mean forecasts against the variability from AMeDAS. The line with the triangles represents the global forecasts, the line with the squares represents the NHM-25km forecasts, and the line with the black dots represents the NHM- $5 \mathrm{~km}$ forecasts.

errors. Figure $5 b$ shows the RMSEs of the ensemble mean forecasts. Using the ensemble means reduces the RMSEs for each resolution. The RMSEs of the NHM- $25 \mathrm{~km}$ and NHM-5km forecasts are an average of $15 \%$ smaller than those of the single forecasts. The improvements of the NHM-25km and NHM- $5 \mathrm{~km}$ forecasts are $10 \%$ greater than those of the global forecasts. This suggests that ensemble downscaling forecasts represent the small-scale uncertainties in addition to the large-scale uncertainties. Consequently, the RMSEs of the ensemble mean NHM- $25 \mathrm{~km}$ and NHM-5km forecasts are comparable to those of the global ensemble mean forecasts. Figure $4 \mathrm{~b}$ shows the ratio of the natural variability of the ensemble mean forecasts to the variability of the AMeDAS observations. The natural variability of the ensemble mean global forecasts is reduced with forecast time. In ensemble mean downscaling forecasts, the small-scale phenomena unforced by the large-scale phenomena must be filtered out with ensemble mean; however, the unforced smallscale phenomena may possibly not be filtered out completely due to the limited ensemble size. Therefore, the natural variability of the ensemble mean downscaling forecasts is composed of those of largescale phenomena and small-scale phenomena forced by the large-scale phenomena. Thus, the natural variability in the ensemble mean downscaling forecasts may be reduced due to the reduction of the natural variability of the global ensemble mean forecasts. While the natural variabilities of the ensemble mean NHM- $25 \mathrm{~km}$ and NHM-5km forecasts are reduced with forecast time, the ensemble mean NHM-25km and NHM-5km forecasts have larger natural variabilies than the global forecasts as shown in Fig. $4 \mathrm{~b}$ These results indicate that the ensemble means of downscaling forecasts can extract more reliable signals including the effects of local circulations.

\subsection{Ensemble spread}

Ensemble spreads ideally should represent the variances of the probability distribution function of atmospheric states (Takano 2002). For probabilistic forecasts, it is important that the ensemble spreads reflect the uncertainties of the forecasts. Figure $5 \mathrm{c}$ shows the spreads of the global, NHM- $25 \mathrm{~km}$, and NHM- $5 \mathrm{~km}$ forecasts. The NHM- $25 \mathrm{~km}$ and NHM- $5 \mathrm{~km}$ forecasts have $80 \%$ larger spreads than those of the global forecasts. Under perfect model assumptions, the expected value of the squared ensemble spread $S$ is

$$
\left\langle S^{2}\right\rangle \equiv\left\langle\frac{1}{M} \sum_{i}^{M}\left(x_{i}-\bar{x}\right)^{2}\right\rangle=\frac{M-1}{2 M}\langle D\rangle,
$$

where angled brackets indicate the expected values, $x_{i}$ and $\bar{x}$ represent the atmospheric states of the $i$-th 

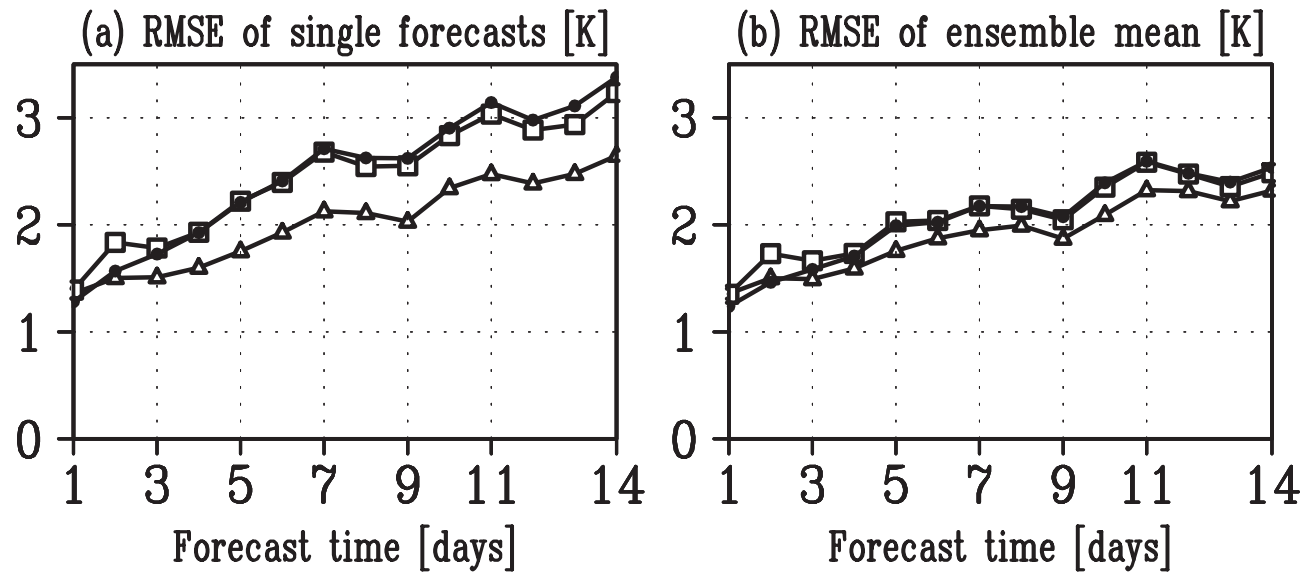

(c) Ensemble spread [K]

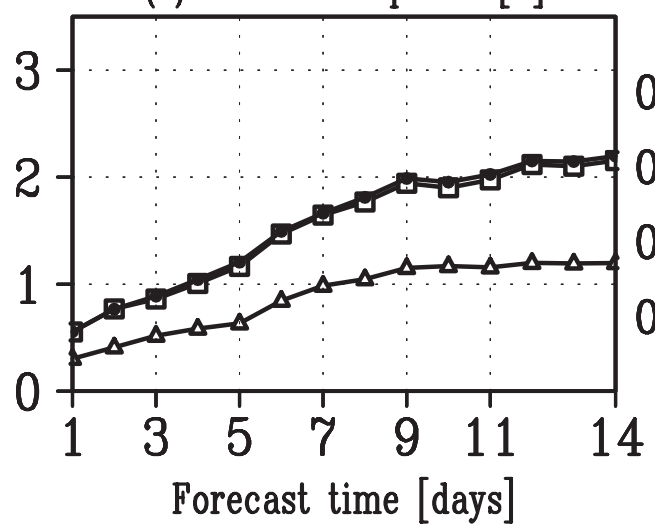

(d) Spread-Skill relationship
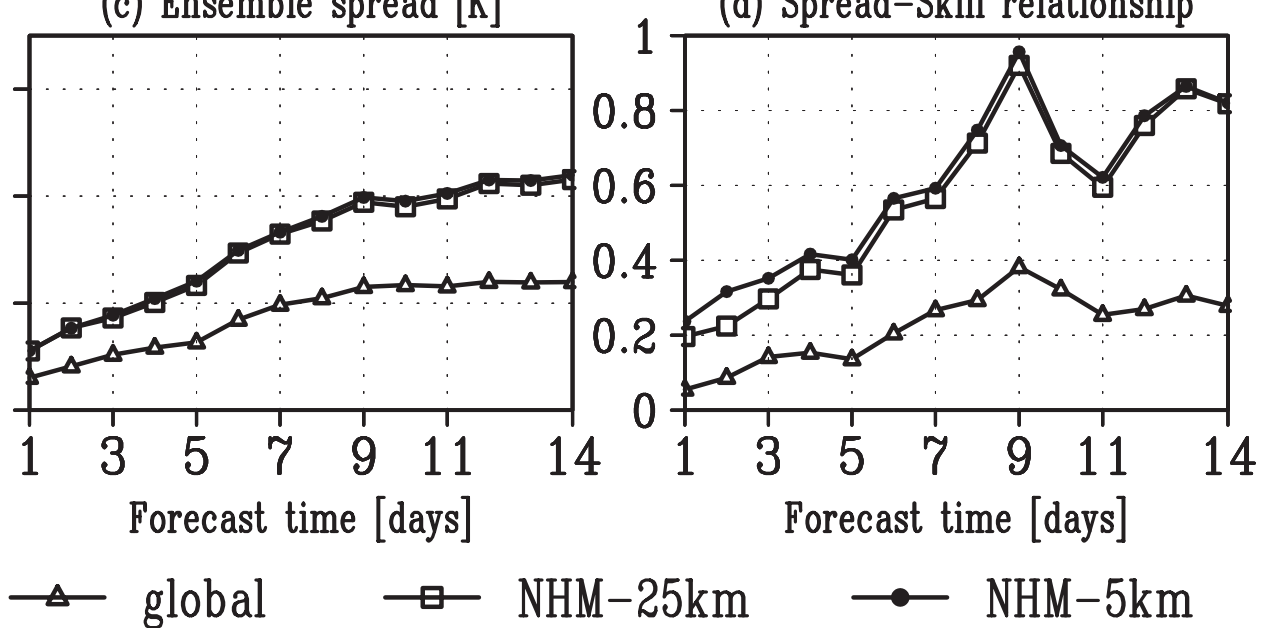

Fig. 5. (a) RMSEs of the daily mean temperature of the single forecasts. (b) RMSEs of the daily mean temperature of the ensemble mean forecasts. (c) Ensemble spreads for the daily mean temperature. (d) Ratio of spreads of RMSEs $(R)$ for the daily mean temperature. The line with the triangles represents the global forecasts, the line with the squares represents the NHM- $25 \mathrm{~km}$ forecasts, and the line with the black dots represents the NHM- $5 \mathrm{~km}$ forecasts.

member and the corresponding ensemble mean, $D$ is the variance of the probability distribution function of atmospheric states, and $M$ is the number of ensemble members. The expected RMSE value of the ensemble mean $E$ is

$$
\left\langle E^{2}\right\rangle=\frac{M+1}{2 M}\langle D\rangle
$$

Thus, the ratio of the spreads to the RMSEs,

$$
R=\frac{M+1\left\langle S^{2}\right\rangle}{M-1\left\langle E^{2}\right\rangle}
$$

should be 1 if the spreads have ideal values (Takano 2002). $R$ indicates whether the spreads can represent the ideal variances. Figure $5 \mathrm{~d}$ shows $R$ of the global, NHM- $25 \mathrm{~km}$, and NHM- $5 \mathrm{~km}$ forecasts. The increase in spreads with downscaling causes the NHM- $25 \mathrm{~km}$ and NHM-5km forecasts to outperform the global forecasts in terms of $R$. This suggests that the spreads of the ensemble downscaling forecasts capture the uncertainties associated with local circulations and implies that the ensemble downscaling forecasts enables probabilistic predictions information about local circulations.

\section{EOF mode decomposition}

Figure 6 shows the modes derived from the EOF analysis of the surface temperatures observed at 

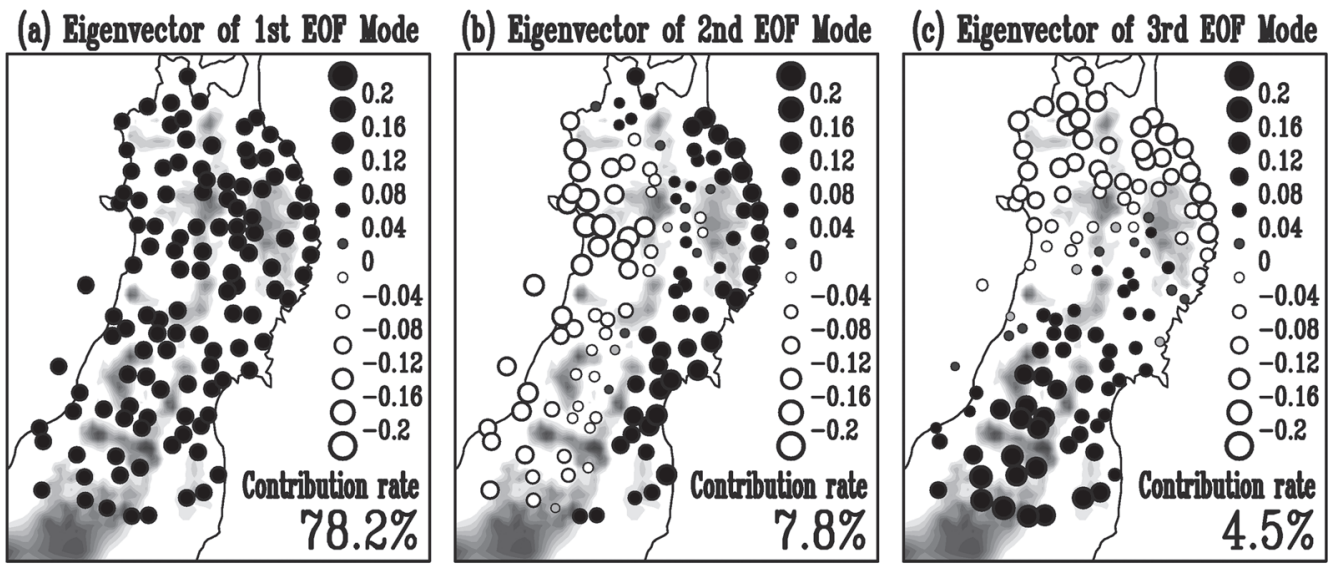

Fig. 6. (a) The first EOF mode, (b) the second EOF mode, and (c) the third EOF mode for the daily mean temperature field observed at the AMeDAS sites.

AMeDAS sites in northeastern Japan. The contributions are $78.2 \%$ for the first mode, $7.8 \%$ for the second mode, and $4.5 \%$ for the third mode. Note that because the contribution of the fourth mode is only $2.0 \%$ we exclude the fourth and higher-order modes from the analysis. These results are consistent with those of Takai et al. (2006), who performed an EOF analysis of AMeDAS temperature data from May to August of 2000-2002. The first mode has the same signs and nearly the same magnitudes at all sites in the domain and indicates the temperature variability over all of northeastern Japan. Figure 7a shows the regression coeffcients of the anomalies of the geopotential height on the $500 \mathrm{hPa}$ surface obtained by JRA-25/JCDAS against the first EOF mode components of the AMeDAS observations and the associated correlation coeffcients. The variation of the first EOF mode is significantly correlated to the intensity of North Pacific anticyclone. The second mode has opposite signs on the Pacific side and the Sea of Japan side that are divided by the mountains that run from north to south over northeastern Japan. This mode represents the temperature field on typical Yamase days as shown in Fig. 1. Figure 7b shows the regression coeffcients of the anomalies of sea level pressure from JRA-25/JCDAS against the second EOF mode components of the AMeDAS observations and the associated correlation coeffcients. The variation of the second EOF mode has a significant correlation with the existence of the Okhotsk high. The second EOF mode is considered to be the Yamase mode. The third mode has opposite signs in the northern and southern parts of the region. This mode may reflect the Baiu front in northeastern Japan.

To determine the predictability of each EOF mode, we compare the decomposed components of the ensemble mean forecasts to the AMeDAS observations. The null hypothesis that there is no correlation between them is rejected with greater than $99 \%$ confidence when the correlation coeffcient is greater than 0.4 . The threshold of predictability is defined as correlation coeffcient of 0.5 . Figure 8 shows the correlation coeffcients of the components of the ensemble mean forecasts versus those of the AMeDAS observations for each mode. The correlation coeffcients indicate that the predictable periods of the forecasts are 8 days for the first mode, 5 days for the Yamase mode (the second mode), and 2 days for the third mode. These results suggest that the predictability largely depends on the EOF modes. Improvement of the correlation coeffcients by dynamical downscaling do not occur except in the short-range forecasts of the Yamase mode and the third mode. Dynamical downscaling does not extend the predictable periods for medium-range forecast.

To assess the extent to which the amplitude of each mode is predictable, we check the regression coeffcients. Figure 9 shows the regression coeffcients of the components of the ensemble mean forecasts against those of the AMeDAS observations for each mode. While the regression coeffcients of the global forecasts for the first mode is approximately 0.7 until day three, those of the NHM- $25 \mathrm{~km}$ and NHM- $5 \mathrm{~km}$ forecasts approach 1 . For the second mode, the regression coeffcients of the global forecasts are less than 0.2 throughout the forecast periods. On the other 
(a) Z500 Correlation (1st mode)

(b) Psea Correlation (2nd mode)
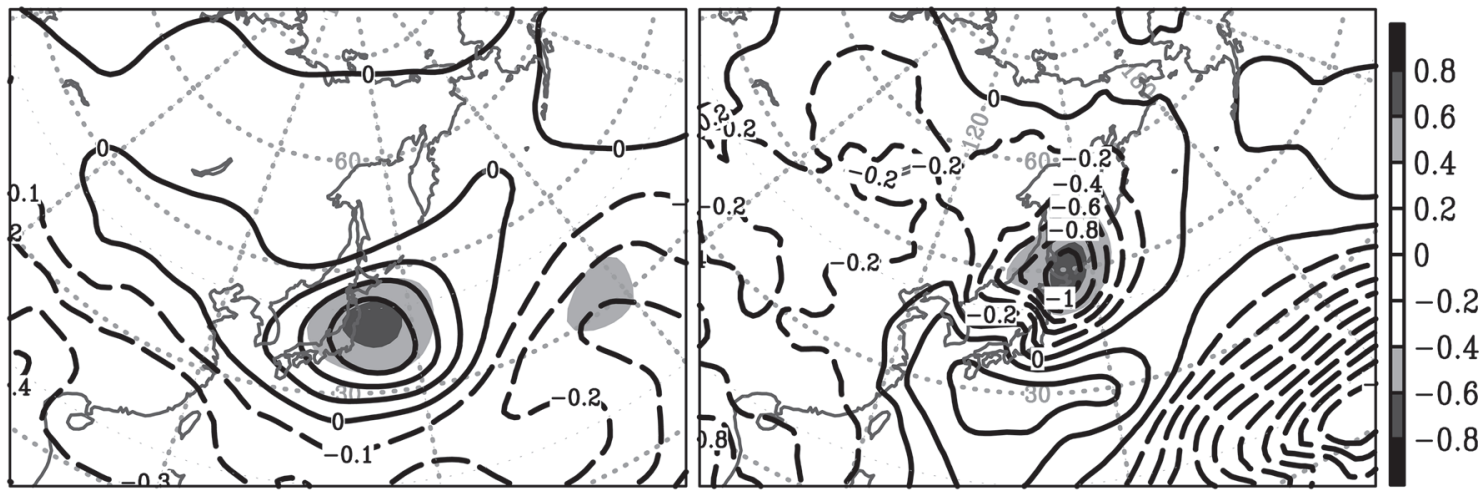

Fig. 7. (a) The regression coeffcients of the anomalies of geopotential height at $500 \mathrm{hPa}$ from JRA-25 (contours) versus the first EOF mode components of the AMeDAS observations and correlation coeffcients between them (shades). (b) The regression coeffcients of the anomalies of sea level pressure from JRA-25 (contours) and correlation coeffcients between them (shades) for the second EOF mode component of the AMeDAS observations.

(a) 1 st mode

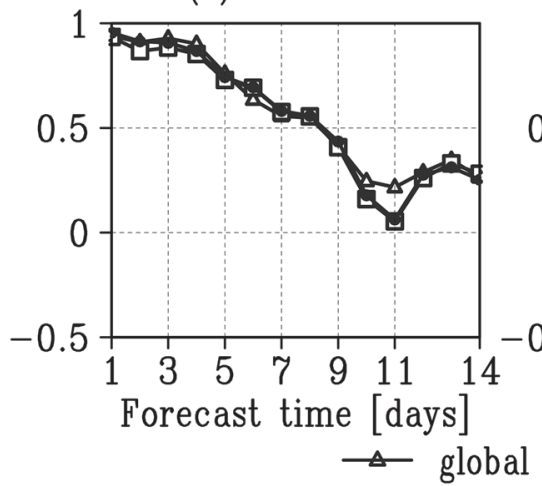

(b) 2nd mode

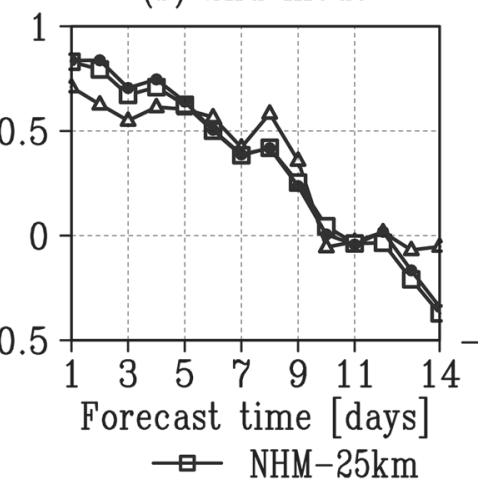

(b) 3rd mode

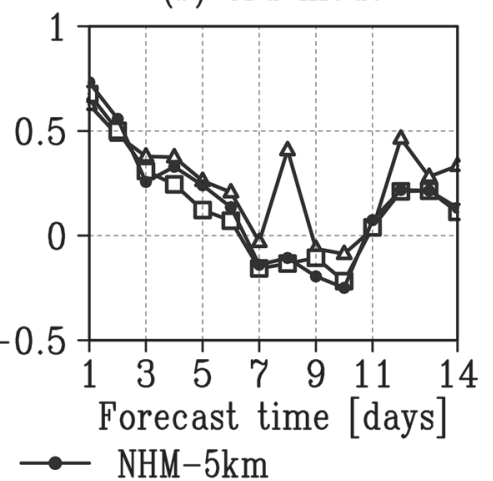

Fig. 8. Correlation coeffcients between the ensemble mean forecasts and AMeDAS for (a) the first EOF mode, (b) the second EOF mode, and (c) the third EOF mode. The line with the triangles represents the global forecasts, the line with the squares represents the NHM-25km forecasts, and the line with the black dots represents the NHM- $5 \mathrm{~km}$ forecasts.

hand, those for the NHM- $25 \mathrm{~km}$ and NHM- $5 \mathrm{~km}$ forecasts are 0.8 and 0.9 , respectively, until day five. For the third mode, the regression coeffcients are 0.2 for the global forecasts and 0.6 for the NHM-25km and NHM- $5 \mathrm{~km}$ forecasts until day two. These results suggest that dynamical downscaling greatly improves the quantitative predictions of the amplitude of the modes. While the NHM-25km forecasts greatly improve the regression coeffcients compared with the global forecasts, a clear improvement is not found in the NHM- $5 \mathrm{~km}$ forecasts compared with the NHM-25km forecasts.

Figure 10 shows the ensemble spreads normalized by the natural variabilities of the global, NHM-25km, and NHM-5km forecasts for each mode. When the value is one, the ensemble members are spread over the same space as the respective model climatology. The normalized spreads are independent of the resolutions. However, the growth of the spreads is slower when the predictability of mode is longer. Indeed, the 
(a) 1st mode

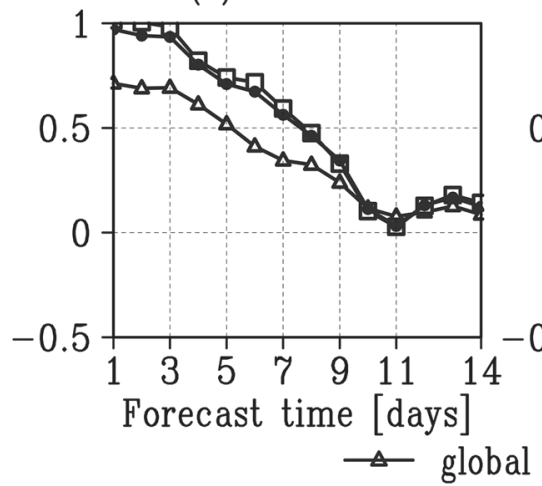

(b) 2nd mode

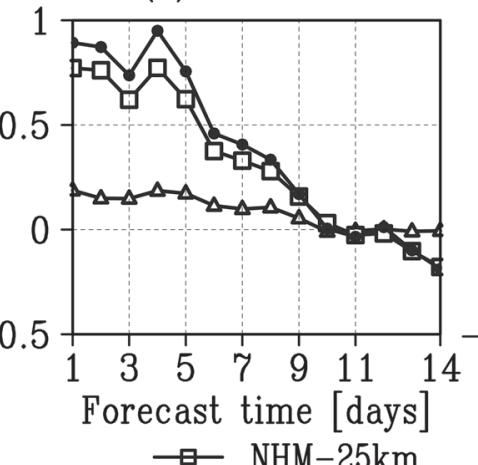

(b) 3rd mode

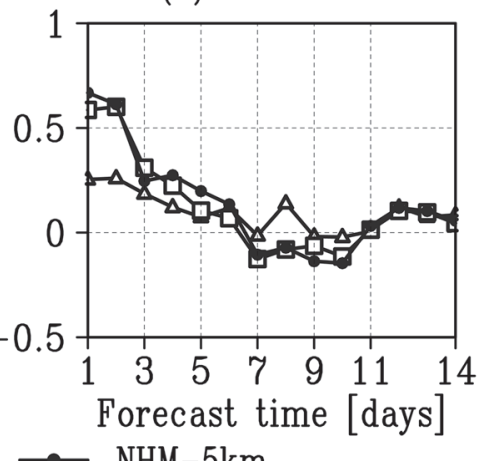

Fig. 9. Regression coeffcients of the ensemble mean forecasts versus the AMeDAS observations for (a) the first EOF mode, (b) the second EOF mode, and (c) third EOF mode. The line with the triangles represents the global forecasts, the line with the squares represents the NHM-25km forecasts, and the line with the black dots represents the NHM-5km forecasts.

(a) 1 st mode

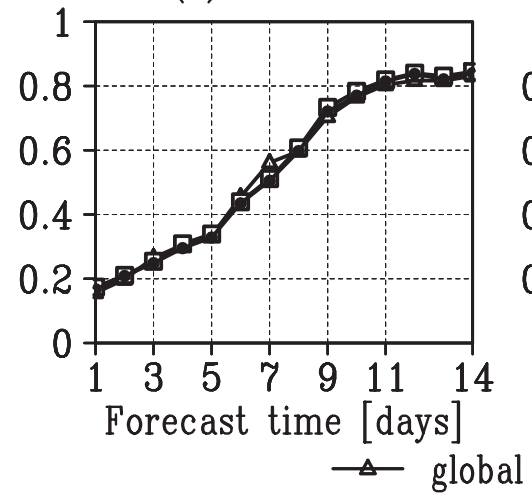

(b) 2nd mode

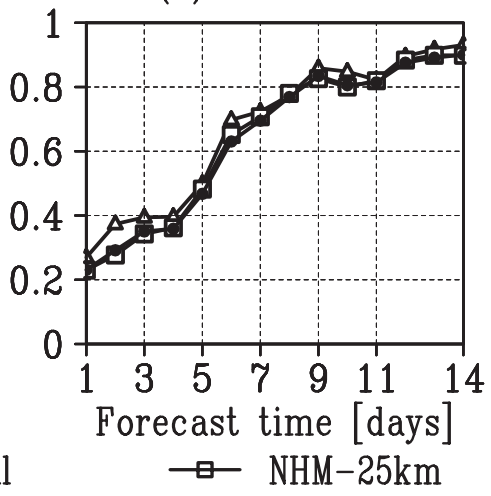

(b) 3rd mode

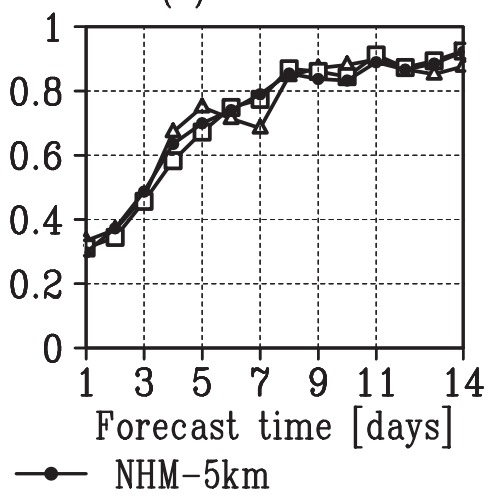

Fig. 10. Ensemble spreads normalised with natural variabilities for (a) the first EOF mode, (b) the second EOF mode, and (c) the third EOF mode. The line with the triangles represents the global forecasts, the line with the squares represents the NHM-25km forecasts, and the line with the black dots represents the NHM-5km forecasts.

times to exceed 0.5 are 8 days for the first mode, 6 days for the Yamase mode, and 4 days for the third mode. This indicates that the ensemble spreads properly reflect the predictability of the modes.

\section{Discussion}

In this section, we discuss what differs the global, NHM-25km, and NHM-5km forecasts.

The amplitude of the Yamase mode is improved in the NHM-25km forecast in comparison with the global forecasts. When Yamase blows to Tohoku district, the cool inflow and accompanying low level clouds are blocked by the mountains on the Pacific coast side. On the other hand, the upper air-mass across the mountains flow along the leeward slopes and it is heated by solar radiation on the Japanese coast side. These processes are essential for the occurrence of the temperature contrast between the Pacific coast side and the Japanese coast side. As a result, the Yamase mode is enhanced. Therefore, in order to reproduce the Yamase mode realistically, it is necessary to resolve the Japanese sea coast, the Pacific coast, and the mountain region elongated north-south over Tohoku district in the models. The global model 
does not have enough resolution. Since the horizontal resolution of the global data is $1.25^{\circ}$, there are only two or three grid points in east-west direction across Tohoku district. The height of the terrain in Tohoku district represented in the global model reaches to less than $220 \mathrm{~m}$. However, NHM-25km and NHM-5km have enough resolution to represent the topographic features over Tohoku district. There are 8 grid points in NHM-25km and 40 grid points in NHM- $5 \mathrm{~km}$ across Tohoku district. The height in the mountain region is depicted higher than $400 \mathrm{~m}$ in NHM-25km and $600 \mathrm{~m}$ in NHM-5km.

The SST data sets for the lower boundary conditions and the parameterization schemes for physical processes also are different between the global and downscaling models as described in Section 2.1. These configurations may improve the performance of the downscaling models to represent meso-scale phenomena. The temperature along the Pacific coast is constrained by the offshore SST when Yamase prevails (Kodama 1997). In order to check the sensitivity to the SST data sets, we conducted an additional downscaling experiment with $25 \mathrm{~km}$ horizontal resolution NHM adopting COBE-SST instead of OISST. The initial times were set as 20 June, 30 June, 10 July, and 20 July in 2003. Note that during these periods Yamase prevailed and caused anomalously cool summer in Tohoku district. The root mean square differences (RMSDs) of the surface temperature between the NHM forecasts with COBE-SST and with OTSST are less than $0.2 \mathrm{~K}$ at most points except a few points along coastal line. The differences are less than $10 \%$ of the RMSDs between the global and the NHM with OISST. Therefore, adopting different SST data sets causes a relatively small impact on the temperature forecasts. The impact of the different cumulus parameterization on the temperature fields is assumed small, because heavy precipitation is rarely observed when Yamase prevails. The representation of cloud-radiation interactions are important to reproduce low-level clouds accompanied by Yamase (Nagasawa et al. 2006). Although the differences in the cloud scheme and time interval to solve the radiative transfer equations might bring some advantages to the NHM forecasts, we could not check the impact because the global data set does not contain the variables associated with radiation process.

Some improvements are also found in the NHM- $5 \mathrm{~km}$ forecasts as compared with the NHM- $25 \mathrm{~km}$ forecasts; however, the improvements are limited. As shown in Fig. 5b, the RMSEs of the NHM-5km forecasts are reduced up to day 3 , wherea a clear differ- ence between the NHM-25km and NHM-5km forecasts is not found after the day 4. For the Yamase mode, the regression coeffcients against the observations improved until day 9 in the NHM- $5 \mathrm{~km}$ forecasts compared to the NHM- $25 \mathrm{~km}$ forecasts as shown in Fig. 9b. Note that the improvement may not be significant beyond the predictable period, considering the value of correlation coeffcients. The improvements depend on the growth of the errors in the lateral boundary conditions. The improvements of the NHM- $5 \mathrm{~km}$ forecasts are enhanced during the periods when the errors in lateral boundary conditions are small, on the other hand, the improvements become reduced if the errors in lateral boundary condtions are grown.

\section{Conclusions}

We assessed medium-range forecasts of the detailed spatial distributions of surface temperature by applying dynamical downscaling with $25 \mathrm{~km}$ and $5 \mathrm{~km}$ resolution regional nonhydrostatic models. The initial and lateral boundary conditions are taken from $1.25^{\circ}$ resolution global ensemble forecasts that consist of nine members. Special attention is paid to the cool summers caused by Yamase, which causes characteristic surface temperature distributions over northeastern Japan. The experimental periods are from late June to early August in 2000-2009, the season in which Yamase occurs most frequently. The bias corrected forecasts of the daily mean surface temperature are verified against the observations at AMeDAS sites.

An ensemble dynamical downscaling forecast system properly reproduces the natural variability of the daily mean temperatures and successfully extracts reliable signals that includes information about local circulations. While the global model simulates $60 \%$ natural variability, the natural variabilities simulated by the downscaling models are comparable to the observed variabilities. The increased natural variability due to dynamical downscaling tends to add small-scale errors in addition to large-scale errors. As a result, the single downscaling forecasts have $20 \%$ larger RMSEs than the global forecasts. However, the ensemble mean forecasts can filter out the unpredictable noises that arise from uncertainties in the lateral boundary conditions. The ensemble mean downscaling forecasts have $15 \%$ smaller RMSEs than the single downscaling forecasts, but they still have much greater natural variabilities than the global forecasts.

Ensemble downscaling also indicates the possibility of probabilistic predictions that consider the effects 
of local circulations. The ensemble downscaling forecasts have $80 \%$ larger spreads than the global forecasts and approach the theoretical value. The spreads in downscaling forecasts include information about the uncertainties of local circulations in addition to largescale motions. This is also a result of the improved reproduction of natural variability.

We decomposed the ensemble mean forecasts of the daily mean surface temperature fields into EOF modes of the temperature fields observed at the AMeDAS sites. The predictability of the daily mean surface temperatures depends significantly on the EOF modes. The predictable periods are 8 days for the homogeneous northeastern Japan mode (the first mode), 5 days for Yamase mode (the second mode), and 2 days for the north-south mode (the third mode).

The decomposition also reveals that dynamical downscaling can properly predict the amplitudes of the modes, although it does not significantly extend the predictable periods. For the Yamase mode, dynamical downscaling can predict $90 \%$ of the observed amplitude, although the global model only predicts 20 $\%$ of the observed amplitude.

The $25 \mathrm{~km}$ horizontal resolution model may be suffcient for medium-range forecast of the daily mean surface temperature. However, it is unclear whether $25 \mathrm{~km}$ horizontal resolution models are suffcient. Yamase usually accompanies low-level clouds that cause fine distributions of diurnal temperature variations. Diurnal temperature variations are of great concern to industries such as agriculture. Therefore, further studies are necessary to investigate the prediction of diurnal temperature variation fields.

\section{Acknowledgments}

The authors thank Climate Prediction Division of Japan Meteorological Agency for providing the one-month ensemble hindcast dataset.

The dynamical downscaling in this study was conducted using Japan Meteorological Agency nonhydrostatic model developed by Meteorological Research Institute and Numerical Prediction Division of Japan Meteorolog- ical Agency.

This study is partially supported by the Japanese Ministry of Education, Culture, Sports, Science and Technology through the Research Program on Climate Change Adaptation (RECCA).

The authors are grateful to two anonymous reviewers for their valuable comments.

\section{References}

Arakawa, A., and W. H. Schubert, 1974: Interaction of a cumulus cloud ensemble with the large-scale environment, part I. J. Atmos. Sci., 31, 674-701.

Buizza, R., and T. N. Palmer, 1995: The singular-vector structure of the atmospheric global circulation. $J$. Atoms. Sci., 52, 1434-1456.

Duchon, C. E., 1979: Lanczos filtering in one and two dimensions. J. Appl. Meteor., 18, 1016-1022.

Hirai, M., T. Sakashita, H. Kitagawa, T. Tsuyuki, M. Hosaki, and M. Oh'izumi, 2007: Development and validation of a new land surface model for JMA's operational global model using the CEOP observation dataset. J. Meteor. Soc. Japan, 85, 1-24.

Ikawa, M., and K. Saito, 1991: Description of a non-hydrostatic model developed at the Forecast Research Department of the MRI. Tech. Rep. MRI, 28, $238 \mathrm{pp.}$

Ishii, M., A. Shouji, S. Sugimoto, and T. Matsumoto, 2005: Objective analyses of sea-surface temperature and marine meteorological variables for the 20th century using ICOADS and the Kobe collection. Int. J. Climatol., 25, 865-879.

Ishizaki, N., I. Takayabu, M. Oh'izumi, H. Sasaki, K. Dairaku, S. Iizuka, F. Kimura, H. Kusaka, S. A. Adachi, K. Kurihara, and K. Tanaka, 2012: Improved performance of simulated Japanese climate with a multi-model ensemble. J. Meteor. Soc. Japan, 90, 235-254.

Iwasaki, T., S. Yamada, and K. Tada, 1989: A parameterization scheme of orographic gravity wave drag with two different vertical partitionings, Part 1: Impact on medium range forecasts. J. Meteor. Soc. Japan, 67, $11-41$.

Japan Meteorological Agency, 2013: Outline of the operational numerical weather prediction at the Japan Meteorological Agency. Appendix to WMO technical progress report on the global data-processing and forecasting system and numerical weather prediction. [Available at http://www.jma.go.jp/jma/jma-eng/jma-center/ nwp/outline2013-nwp/index.htm.]

Kain, J. S., and J. M. Fritsch, 1990: A one-dimensional entraining/detraining plume model and its application in convective parameterization. J. Atmos. Sci., 47, 2784-2802.

Kanno, H., 1993: Differences of temperature and humidity between Yamase and sea breeze and their seasonal variations at Hachinohe in Aomori prefecture. Tenki, 40, 751-757 (in Japanese).

Kawai, H., and T. Inoue, 2006: A simple parameterization scheme for subtropical marine stratocumulus. SOLA, 2, 17-20.

Kida, H., T. Koide, H. Sasaki, and M. Chiba, 1991: A new approach for coupling a limited area model to a GCM for regional climate simulations. J. Meteor. Soc. Japan, 69, 723-728.

Kodama, Y., 1997: Airmass transformation of the Yamase air-flow in the summer of 1993. J. Meteor. Soc. Japan, 75, 737-751. 
Marsigli, C., A. Montani, F. Nerozzi, T. Paccagnella, S. Tibaldi, F. Molteni, and R. Buizza, 2001: A strategy for high-resolution ensemble prediction. II: Limitedarea experiments in four Alpine flood events. Quart. J. Roy. Meteor. Soc., 127, 2095-2115.

Marsigli, C., A. Montani, and T. Paccagnella, 2008: A spatial verification method applied to the evaluation high-resolution ensemble forecasts. Meteor. Appl., 15, 125-143.

Mellor, G. L., and T. Yamada, 1974: A hierarchy of turbulence closure models for planetary boundary layers. $J$. Atmos. Sci., 31, 1791- 1806.

Nagasawa, R., T. Iwasaki, S. Asano, K. Saito, and H. Okamoto, 2006: Resolution dependence of nonhydrostatic models in simulating the formation and evolution of low-level clouds during a "Yamase" event. $J$. Meteor. Soc. Japan, 84, 969-987.

Nakanishi, M., and H. Niino, 2004: An improved Mellor-Yamada level 3 model with condensation physics: Its design and verification. Bound-Layer Meteor., 112, 1-31.

Nakanishi, M., and H. Niino, 2006: An improved Mellor-Yamada level 3 model: Its numerical stability and application to a regional prediction of advection fog. Bound.-Layer Meteor, 119, 397-407.

Ninomiya, K., and H. Mizuno, 1985a: Anomalously cold spell in summer over North-eastern Japan caused by northeasterly wind from polar maritime air-mass. Part 1. EOF analysis of temperature variation in relation to the large-scale situation causing the cold summer. $J$. Meteor. Soc. Japan, 63, 845-857.

Ninomiya, K., and H. Mizuno, 1985b: Anomalously cold spell in summer over North-eastern Japan caused by northeasterly wind from polar maritime air-mass. Part 2. Structure of the north-easterly flow from polar maritime airmass. J. Meteor. Soc. Japan, 63, 859-871.

Onogi, K., J. Tsutsui, H. Koide, M. Sakamoto, S. Kobayashi, H. Hatsushika, T. Matsumoto, N. Yamazaki, H. Kamahori, K. Takahashi, S. Kadokura, K. Wada, K. Kato, R. Oyama, T. Ose, N. Mannoji, and R. Taira, 2007: The JRA-25 reanalysis. J. Meteor. Soc. Japan, 85, 369-432.

Reynolds, R. W., T. M. Smith, C. Liu, D. B. Chelton, K. S. Casey, and M. G. Schlax, 2007: Daily high-resolution-blended analyses for sea surface temperature. $J$. Climate, 20, 5473-5496.

Saito, K., J. Ishida, K. Aranami, T. Hara, T. Segawa, M. Narita, and Y. Honda, 2007: Nonhydrostatic atmospheric models and operational development at JMA. J. Meteor. Soc. Japan, 85, 271-304.

Sommeria, G., and J. W. Deardorff, 1977: Subgrid-scale condensation in models of nonprecipitating clouds. $J$. Atmos. Sci., 34, 344-355.

Takai, H., H. Kawamura, and O. Isoguchi, 2006: Characteristics of the Yamase winds over oceans around Japan observed by the scatterometer-derived ocean surface vector winds. J. Meteor. Soc. Japan, 84, 365- 373.

Takano, K., 2002: The application technique of ensemble forecasts. Meteor. Res. Note, 201, 73-103 (in Japanese).

Toth, Z., and E. Kalnay, 1993: Ensemble forecasting at NMC: The generation of perturbations. Bull. Amer. Meteor. Soc., 74, 2317-2330.

Toth, Z., and E. Kalnay, 1997: Ensemble forecasting at NCEP and the breeding method. Mon. Wea. Rev., 125, 3297-3319.

Vukicevic, T., and R. M. Errico, 1990: The influence of artificial and physical factors upon predictability estimates using a complex limited area model. Mon. Wea. Rev., 118, 1460-1482.

Wei, M., Z. Toth, R. Wobus, and Y. Zhu, 2008: Initial perturbations based on the ensemble transform (ET) technique in the NCEP global operational forecast system. Tellus A, 60, 62-79.

Yasunaga, K., H. Sasaki, Y. Wakazuki, T. Kato, C. Muroi, A. Hashimoto, S. Kanada, K. Kurihara, M. Yoshizaki, and Y. Sato, 2005: Performance of long-term integration of the Japan Meteorological Agency nonhydrostatic model using the spectral boundary coupling method. Wea. Forecasting, 20, 1061-1072. 